\title{
Determinantes sociales de la salud asociados a la severidad y mortalidad en pacientes con COVID-19
}

\author{
Rodrigo Núñez-Cortés, ${ }^{1 *}$ Maritza Ortega-Palavecinos, ${ }^{2}$ Camilo Soto-Carmona, ${ }^{3}$ Pablo Torres-Gangas, ${ }^{3}$ \\ Ma. Paz Concha-Rivero 4 y Rodrigo Torres-Castro ${ }^{1}$ \\ ${ }^{1}$ Universidad de Chile, Facultad de Medicina, Departamento de Terapia Física; ${ }^{2}$ Hospital Clínico La Florida, Servicio Social; ${ }^{3} \mathrm{Hospital}$ Clínico La \\ Florida, Servicio de Terapia Física; ${ }^{4}$ Hospital Clínico La Florida, Servicio de Enfermería. Santiago, Chile
}

\section{Resumen}

Introducción: Históricamente, las pandemias han tenido como resultado tasas de mortalidad más altas en las poblaciones más vulnerables. Los determinantes sociales de la salud (DSS) se han asociado a la morbimortalidad de las personas en diferentes niveles. Objetivo: Determinar la relación entre los DSS, la severidad de COVID-19 y la mortalidad por esta enfermedad. Métodos: Estudio retrospectivo en el que se recolectaron datos de pacientes con COVID-19 en un hospital público de Chile. Las variables sociodemográficas relacionadas con los DSS estructurales se clasificaron según las siguientes categorías: sexo, edad (< 65 años, $\geq 65$ años), educación secundaria (completada o no), condición de trabajo (activo, inactivo) e ingreso económico (< USD 320, $\geq$ USD 320). Resultados: Fueron incluidos 1012 casos con COVID-19 confirmados por laboratorio. La edad promedio fue de $64.2 \pm 17.5$ años. La mortalidad de la muestra total fue de $14.5 \%$. La edad, nivel educativo, desempleo e ingresos tuvieron fuerte asociación con la mortalidad $(p<0.001)$. Conclusiones: Los hallazgos refuerzan la idea de que los DSS deben considerarse una prioridad de salud pública, por lo que los esfuerzos políticos deben centrarse en reducir las desigualdades en salud para las generaciones futuras.

PALABRAS CLAVE: Determinantes sociales de la salud. Síndrome respiratorio agudo grave. Coronavirus. Mortalidad.

\section{Social determinants of health associated with severity and mortality in patients with COVID-19}

\begin{abstract}
Introduction: Historically, pandemics have resulted in higher mortality rates in the most vulnerable populations. Social determinants of health (SDH) have been associated with people morbidity and mortality at different levels. Objective: To determine the relationship between SDH and COVID-19 severity and mortality. Methods: Retrospective study, where data from patients with COVID-19 were collected at a public hospital in Chile. Sociodemographic variables related to structural SDH were classified according to the following categories: gender, age (<65 years, $\geq 65$ years), secondary education (completed or not), work status (active, inactive) and income (< USD 320, $\geq$ USD 320). Results: A total of 1,012 laboratory-confirmed COVID-19 cases were included. Average age was $64.2 \pm 17.5$ years. Mortality of the entire sample was $14.5 \%$. Age, level of education, unemployment and income had a strong association with mortality $(p<0.001)$. Conclusions: The findings reinforce the idea that SDH should be considered a public health priority, which is why political efforts should focus on reducing health inequalities for future generations.
\end{abstract}

KEY WORDS: Social determinants of health. Severe acute respiratory syndrome. Coronavirus. Mortality.

Correspondencia:

*Rodrigo Núñez-Cortés

E-mail: r_nunez@uchile.cl
Gac Med Mex. 2021;157:273-280

Disponible en PubMed

www.gacetamedicademexico.com CC BY-NC-ND (http://creativecommons.org/licenses/by-nc-nd/4.0/). 


\section{Introducción}

En diciembre de 2019 se produjo un brote de neumonía de etiología desconocida en Wuhan, China, que resultó en el aislamiento de una nueva cepa de coronavirus. La Organización Mundial de la Salud nombró SARS-CoV-2 a ese virus, ${ }^{1}$ el cual evolucionó a pandemia en pocas semanas. ${ }^{2}$ La presentación clínica de COVID-19 comprende principalmente fiebre, tos, disnea y compromiso del parénquima pulmonar que, en algunos casos, progresa a síndrome respiratorio agudo severo, necesidad de ingreso a unidades de cuidados intensivos ( $\mathrm{UCl}) \mathrm{y}$, eventualmente, produce la muerte. ${ }^{3}$

Los pacientes se clasifican con COVID-19 grave o no grave según la directrices establecidas por la Sociedad Americana de Tórax y la Sociedad Americana de Enfermedades Infecciosas. ${ }^{4}$ Los criterios de admisión a la UCI incluyen ventilación mecánica invasiva o choque séptico que hace necesaria la administración de vasopresores. ${ }^{5}$ Aunque hay factores de riesgo conocidos para el agravamiento de la enfermedad (por ejemplo, comorbilidades), ${ }^{1}$ numerosos autores han propuesto la necesidad de considerar la implicación de los determinantes sociales en este gran desafío para la salud pública. ${ }^{6,7}$

Los determinantes sociales de la salud (DSS) son las condiciones en que las personas nacen, crecen, trabajan, viven y envejecen, incluyendo el conjunto más amplio de fuerzas y sistemas que influyen sobre las condiciones de la vida diaria, ${ }^{8}$ las relaciones con el sistema político, normas sociales y consideraciones ambientales. ${ }^{9,10}$ Los DSS se han asociado en diferentes niveles a la morbimortalidad. ${ }^{11}$ Por ejemplo, la mortalidad en adultos mayores se asocia a dificultades económicas, nivel de escolaridad, participación social, actividad física y tabaquismo, entre otros. ${ }^{12} \mathrm{El}$ nivel socioeconómico, representado por el nivel educativo, el ingreso y la ocupación, ${ }^{13}$ también se asocia a mayor mortalidad y menor expectativa de vida en diferentes países y grupos étnicos. ${ }^{13,14}$ Además, los DSS han cumplido un papel importante en el surgimiento y propagación de otras pandemias, como la ocasionada por el virus de la influenza. ${ }^{15}$

Aproximadamente entre 15 y $20 \%$ de los pacientes con COVID-19 desarrollan el estadio grave de la enfermedad y requieren hospitalización. ${ }^{16}$ Este grupo comprende particularmente a adultos mayores y personas con comorbilidades. Existe una fuerte asociación entre gravedad, mortalidad de la enfermedad y comorbilidades cardiometabólicas como la obesidad, diabetes o hipertensión. ${ }^{17}$ Estas enfermedades están influidas por causas multifactoriales (factores individuales, sociales y ambientales). Si bien algunos factores biológicos son esenciales para el desarrollo de estos padecimientos, los componentes culturales y sociales, junto con factores socioeconómicos como la profesión e ingreso económico, contribuyen al desarrollo de comportamientos sociales que también son cruciales en su aparición. ${ }^{18}$

Históricamente, las pandemias provocan tasas de mortalidad más altas en poblaciones más vulnerables (por ejemplo, la pandemia de influenza entre 1918 y 1919). ${ }^{19}$ Con la pandemia de COVID-19 es fundamental comprender los factores sociales que determinan un pronóstico grave y de esta manera desarrollar intervenciones tempranas y estrategias de control. El objetivo de este estudio fue determinar la relación entre los DSS y el nivel de severidad y mortalidad causadas por COVID-19. Un objetivo secundario fue identificar la relación entre las comorbilidades subyacentes y la mortalidad y gravedad causadas por COVID-19.

\section{Métodos}

En este estudio retrospectivo se recopilaron datos de pacientes con COVID-19 en un hospital público de Chile; se revisó la historia clínica de todos los casos confirmados por laboratorio en el Hospital Clínico La Florida (Santiago, Chile) entre el 1 de abril y el 10 de junio de 2020 (10 semanas). Los criterios de inclusión fueron los siguientes: edad $\geq 18$ años, pacientes ambulatorios y hospitalizados con resultado positivo en la prueba de PCR de muestras nasales y faríngeas. ${ }^{20}$ No hubo criterios de exclusión. Este estudio fue aprobado por el comité de ética de la institución. La investigación se realizó de conformidad con los principios éticos de la Declaración de Helsinki. Se renunció al consentimiento informado por escrito debido a la naturaleza retrospectiva del estudio y a la necesidad urgente de recopilar datos sobre COVID-19.

Los seis investigadores de este estudio acordaron el diseñó un formulario de extracción de datos. Los datos recopilados incluyeron antecedentes médicos, antecedentes de exposición y comorbilidades subyacentes. La variable principal de estudio fue la mortalidad y severidad. Se definieron como severos, los casos ingresados en la $\mathrm{UCl}$ debido a insuficiencia respiratoria por la que se requirió ventilación 
mecánica o debido a choque séptico que hizo necesaria la administración de vasopresores. ${ }^{5}$ Las variables sociodemográficas relacionadas con los DSS estructurales se clasificaron según las siguientes categorías: sexo (masculino, femenino), edad $(<65$ años, $\geq 65$ años), educación secundaria (completada o no), condición de trabajo (activo, inactivo) e ingresos económicos (< USD 320, $\geq$ USD 320). Los otros DSS considerados fueron el estado de discapacidad (mental, intelectual o sensorial) y la categoría de adulto institucionalizado. Los resultados clínicos se monitorizaron hasta el 18 de julio de 2020, fecha final de seguimiento (seis semanas después de la admisión del último paciente).

Todos los análisis estadísticos se realizaron en SPSS versión 22.0 (IBM Corporation, Armonk, Nueva York). La normalidad de los datos se verificó mediante la prueba de Shapiro-Wilk. Se calcularon las medias y desviaciones estándar de las variables cuantitativas y los porcentajes de las variables categóricas. La prueba de chi cuadrada sirvió para comparar las variables categóricas. La asociación entre la mortalidad y gravedad de COVID-19 y los DSS se determinó mediante la razón de momios (RM), con un intervalo de confianza de $95 \%$ (IC $95 \%$ ). La significación estadística se estableció en $p<0.05$. Los tamaños del efecto se caracterizaron como pequeño, moderado 0 grande y se establecieron mediante RM de 1.68, 3.47 y 6.71 , respectivamente. ${ }^{21}$

\section{Resultados}

En este estudio fueron incluidos 1012 casos con COVID-19 confirmados por laboratorio. La edad promedio fue de $64.2 \pm 17.5$ años. Un total de 364 (36\%) pacientes fueron hospitalizados y 70 pacientes $(6.9 \%)$ ingresaron a la $\mathrm{UCl}$. La mortalidad de la muestra total fue de $14.5 \%$, mientras que la mortalidad de los casos hospitalizados fue de $39.3 \%$. En la Tabla 1 se describe la caracterización basal de la muestra total.

Se encontró una asociación significativa entre los DSS y la mortalidad y gravedad de COVID-19. En relación con la mortalidad, se observó efecto grande de la edad $\geq 65$ años (RM $=24$, IC $95 \%=14.7-39.2$, $\mathrm{p}<0.001)$, nivel educacional $(\mathrm{RM}=9.6, \mathrm{IC}$ $95 \%=4.6-20.3, p<0.001)$, condición de trabajo inactivo (RM $=16.3, \mathrm{IC} 95 \%=9.8-27.3, \mathrm{p}<0.001) \mathrm{y}$ menores ingresos económicos $(\mathrm{RM}=8.5, \mathrm{IC}$ $95 \%=5.1-14.4, p<0.001)$. Un análisis ajustado por edad mantuvo asociación significativa con la inactividad laboral (RM $=6.1, \mathrm{IC} 95 \%=2.2-16.8, \mathrm{p}<0.001)$
Tabla 1. Características basales de la muestra total $(n=1012)$

\begin{tabular}{|l|c|c|}
\hline Edad media \pm DE & \multicolumn{2}{|c|}{$64.2 \pm 17.5$} \\
\hline Grupo de edad & n & \\
\hline$\geq 65$ años & & 29.5 \\
\hline$<65$ años & 299 & 70.5 \\
\hline Sexo & 713 & \\
\hline Masculino & & 43.4 \\
\hline Femenino & 439 & 56.6 \\
\hline Hospitalizados & 573 & 36 \\
\hline Fallecidos & 364 & 14.5 \\
\hline Admisión a UCl & 147 & 6.9 \\
\hline Ventilación invasiva & 70 & 8.5 \\
\hline Traqueostomía & 86 & 1.4 \\
\hline
\end{tabular}

$\mathrm{DE}$ = desviación estándar, $\mathrm{UCl}=$ unidad de cuidados intensivos.

y menores ingresos económicos (RM $=6.8$, IC $95 \%$ $=1.9-23.9, p=0.001)$. El estado de discapacidad también mostró efecto grande asociado a la mortalidad $(\mathrm{RM}=8.3$, IC $95 \%=4.7-14.4, \mathrm{p}<0.001)$. En cuanto a la severidad, se observó una medida de efecto grande del nivel educacional $(\mathrm{RM}=9.4$, IC $95 \%=4.4-20.3, p<0.001$ ).

Las comorbilidades más prevalentes en los pacientes fallecidos fueron hipertensión (78.9\%), diabetes (44.9\%) y enfermedades cardiovasculares $(24.5 \%)$. Se observó una medida de efecto grande para hipertensión (RM $=10.7$, IC $95 \%=7.0-16.4, p=0.001)$ y enfermedades cerebrovasculares $(\mathrm{RM}=8.2$, IC $95 \%=4.5-15, p=0.001$ ). En la Tabla 2 se detalla la asociación entre DSS, comorbilidades y mortalidad y en la Tabla 3, la asociación entre DSS, comorbilidades y gravedad de la enfermedad.

\section{Discusión}

El objetivo de este estudio fue determinar la relación entre DSS, mortalidad y gravedad de COVID-19. En nuestra muestra, observamos una tasa de mortalidad de $14.5 \%$ en todos los pacientes y de $39.3 \%$ en los casos hospitalizados, mucho mayor que la informada en otros estudios. ${ }^{22,23}$ Los DSS estructurales presentaron una fuerte asociación con la mortalidad, incluidos la edad, el nivel educacional, el desempleo y el ingreso económico. Estos DSS también mostraron 
Tabla 2. Determinantes sociales de la salud y comorbilidades de pacientes con COVID-19 agrupados según el desenlace clínico

\begin{tabular}{|c|c|c|c|c|c|c|c|}
\hline \multirow[t]{2}{*}{ Características } & \multicolumn{2}{|c|}{$\begin{array}{l}\text { Fallecidos } \\
(n=147)\end{array}$} & \multicolumn{2}{|c|}{$\begin{array}{l}\text { No fallecidos } \\
\quad(n=865)\end{array}$} & \multirow[t]{2}{*}{ RM (IC $95 \%)$} & \multirow[t]{2}{*}{ p } & - \\
\hline & $\mathrm{n}$ & $\%$ & $\mathrm{n}$ & $\%$ & & & i \\
\hline $\begin{array}{l}\text { Sexo } \\
\text { Masculino } \\
\text { Femenino }\end{array}$ & $\begin{array}{l}80 \\
67\end{array}$ & $\begin{array}{l}54.4 \\
45.6\end{array}$ & $\begin{array}{l}359 \\
506\end{array}$ & $\begin{array}{l}41.5 \\
58.5\end{array}$ & $1.68(1.18-2.39)$ & 0.002 & 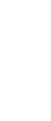 \\
\hline $\begin{array}{l}\text { Edad } \\
\qquad 65 \text { años } \\
<65 \text { años }\end{array}$ & $\begin{array}{c}126 \\
21\end{array}$ & $\begin{array}{l}85.7 \\
14.3\end{array}$ & $\begin{array}{l}173 \\
692\end{array}$ & $\begin{array}{l}20 \\
80\end{array}$ & 24 (14.68-39.22) & $<0.001$ & (). \\
\hline $\begin{array}{l}\text { Educación secundaria } \\
\text { Incompleta } \\
\text { Completa } \\
\text { Desconocida }\end{array}$ & $\begin{array}{l}90 \\
18 \\
39\end{array}$ & $\begin{array}{l}61.2 \\
12.2 \\
26.5\end{array}$ & $\begin{array}{c}272 \\
524 \\
69\end{array}$ & $\begin{array}{c}31.4 \\
60.6 \\
8\end{array}$ & $9.63(4.57-20.29)$ & $<0.001$ & 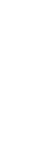 \\
\hline $\begin{array}{l}\text { Condición de trabajo } \\
\text { Inactivo } \\
\text { Activo }\end{array}$ & $\begin{array}{c}129 \\
18\end{array}$ & $\begin{array}{l}87.8 \\
12.2\end{array}$ & $\begin{array}{l}264 \\
601\end{array}$ & $\begin{array}{l}30.5 \\
69.5\end{array}$ & $16.32(9.75-27.28)$ & $<0.001$ & 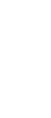 \\
\hline $\begin{array}{l}\text { Ingresos económicos } \\
<\text { USD } 320 \\
\geq \text { USD } 320\end{array}$ & $\begin{array}{c}130 \\
17\end{array}$ & $\begin{array}{l}88.4 \\
11.6\end{array}$ & $\begin{array}{l}409 \\
456\end{array}$ & $\begin{array}{l}47.3 \\
52.7\end{array}$ & $8.5(5.05-14.38)$ & $<0.001$ & $\mid \begin{array}{l}2 \\
0 \\
\frac{0}{5} \\
0 \\
. \subseteq \\
\end{array}$ \\
\hline $\begin{array}{l}\text { Adultos institucionalizados } \\
\text { Sí } \\
\text { No }\end{array}$ & $\begin{array}{c}13 \\
134\end{array}$ & $\begin{array}{c}8.8 \\
91.2\end{array}$ & $\begin{array}{c}21 \\
844\end{array}$ & $\begin{array}{c}2.4 \\
97.6\end{array}$ & $3.89(1.91-7.97)$ & $<0.001$ & 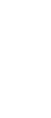 \\
\hline $\begin{array}{l}\text { Sítuación de discapacidad } \\
\text { Sí } \\
\text { No }\end{array}$ & $\begin{array}{c}31 \\
116\end{array}$ & $\begin{array}{l}21.1 \\
78.9\end{array}$ & $\begin{array}{c}27 \\
838\end{array}$ & $\begin{array}{c}3.1 \\
96.9\end{array}$ & $8.29(4.77-14.39)$ & $<0.001$ & 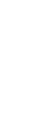 \\
\hline $\begin{array}{l}\text { Historia de tabaquismo } \\
\text { Sí } \\
\text { No }\end{array}$ & $\begin{array}{c}9 \\
138\end{array}$ & $\begin{array}{c}6.1 \\
93.9\end{array}$ & $\begin{array}{c}89 \\
776\end{array}$ & $\begin{array}{c}1 \\
0.3 \\
89.7\end{array}$ & $0.569(0.28-1.16)$ & 0.071 & $\mid$\begin{tabular}{l|}
$\cdot \frac{1}{3}$ \\
$\frac{3}{0}$ \\
$\frac{0}{2}$ \\
$\frac{0}{2}$
\end{tabular} \\
\hline $\begin{array}{l}\text { Alcoholismo } \\
\text { Sí } \\
\text { No }\end{array}$ & $\begin{array}{c}7 \\
140\end{array}$ & $\begin{array}{c}4.8 \\
95.2\end{array}$ & $\begin{array}{c}45 \\
820\end{array}$ & $\begin{array}{c}5.2 \\
94.8\end{array}$ & $0.91(0.4-2.06)$ & 0.509 & $\mid \begin{array}{c}-\frac{1}{2} \\
\frac{-\pi}{0} \\
0 \\
0 \\
0\end{array}$ \\
\hline $\begin{array}{l}\text { Obesidad } \\
\text { Sí } \\
\text { No }\end{array}$ & $\begin{array}{c}19 \\
128\end{array}$ & $\begin{array}{l}12.9 \\
87.1\end{array}$ & $\begin{array}{c}79 \\
786\end{array}$ & $\begin{array}{c}9.1 \\
90.1\end{array}$ & $1.47(0.86-2.52)$ & 0.102 & 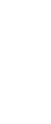 \\
\hline $\begin{array}{l}\text { Hipertensión arterial } \\
\text { Sí } \\
\text { No }\end{array}$ & $\begin{array}{c}116 \\
31\end{array}$ & $\begin{array}{l}78.9 \\
21.1\end{array}$ & $\begin{array}{l}224 \\
641\end{array}$ & $\begin{array}{l}25.9 \\
74.1\end{array}$ & $10.71(7.0-16.37)$ & $<0.001$ & 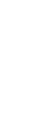 \\
\hline $\begin{array}{l}\text { Diabetes } \\
\text { Sí } \\
\text { No }\end{array}$ & $\begin{array}{l}66 \\
81\end{array}$ & $\begin{array}{l}44.9 \\
55.1\end{array}$ & $\begin{array}{l}137 \\
728\end{array}$ & $\begin{array}{l}15.8 \\
84.2\end{array}$ & $4.33(2.98-6.28)$ & $<0.001$ & $\frac{\bar{c}}{2}$ \\
\hline $\begin{array}{l}\text { Enfermedad cardiovascular } \\
\text { Sí } \\
\text { No }\end{array}$ & $\begin{array}{c}36 \\
111\end{array}$ & $\begin{array}{l}24.5 \\
75.5\end{array}$ & $\begin{array}{c}53 \\
812\end{array}$ & $\begin{array}{c}6.1 \\
93.9\end{array}$ & $4.96(3.11-7.93)$ & $<0.001$ & 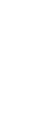 \\
\hline $\begin{array}{l}\text { Enfermedad cerebrovascular } \\
\text { Sí } \\
\text { No }\end{array}$ & $\begin{array}{c}26 \\
121\end{array}$ & $\begin{array}{l}17.7 \\
82.3\end{array}$ & $\begin{array}{c}22 \\
843\end{array}$ & $\begin{array}{c}2.5 \\
95.5\end{array}$ & $8.23(4.52-14.98)$ & $<0.001$ & 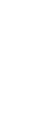 \\
\hline $\begin{array}{l}\text { EPOC } \\
\text { Sí } \\
\text { No }\end{array}$ & $\begin{array}{c}17 \\
130\end{array}$ & $\begin{array}{l}11.6 \\
88.4\end{array}$ & $\begin{array}{c}23 \\
842\end{array}$ & $\begin{array}{c}2.7 \\
97.3\end{array}$ & $4.78(2.49-9.2)$ & $<0.001$ & 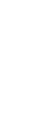 \\
\hline $\begin{array}{l}\text { Otras enfermedades } \\
\text { pulmonares } \\
\text { Sí } \\
\text { No }\end{array}$ & $\begin{array}{c}18 \\
129\end{array}$ & $\begin{array}{l}12.2 \\
87.8\end{array}$ & $\begin{array}{c}46 \\
819\end{array}$ & $\begin{array}{c}5.3 \\
94.7\end{array}$ & $2.48(1.39-4.41)$ & 0.03 & 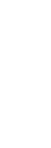 \\
\hline
\end{tabular}


Tabla 2. Determinantes sociales de la salud y comorbilidades de pacientes con COVID-19 agrupados según el desenlace clínico (Continuación)

\begin{tabular}{|c|c|c|c|c|c|c|}
\hline \multirow[t]{2}{*}{ Características } & \multicolumn{2}{|c|}{$\begin{array}{l}\text { Fallecidos } \\
(n=147)\end{array}$} & \multicolumn{2}{|c|}{$\begin{array}{l}\text { No fallecidos } \\
\quad(n=865)\end{array}$} & \multirow[t]{2}{*}{ RM (IC $95 \%$ ) } & \multirow[t]{2}{*}{$p$} \\
\hline & $n$ & $\%$ & $n$ & $\%$ & & \\
\hline $\begin{array}{l}\text { Enfermedad rena } \\
\text { Sí } \\
\text { No }\end{array}$ & $\begin{array}{c}21 \\
126\end{array}$ & $\begin{array}{l}14.3 \\
85.7\end{array}$ & $\begin{array}{c}29 \\
836\end{array}$ & $\begin{array}{c}3.4 \\
96.6\end{array}$ & $4.81(2.65-8.68)$ & $<0.001$ \\
\hline $\begin{array}{l}\text { Cáncer } \\
\text { Sí } \\
\text { No }\end{array}$ & $\begin{array}{c}9 \\
138\end{array}$ & $\begin{array}{c}6.1 \\
93.9\end{array}$ & $\begin{array}{c}20 \\
845\end{array}$ & 97.72 .3 & $2.75(1.23-6.18)$ & 0.017 \\
\hline $\begin{array}{l}\text { Inmunodeficienci } \\
\text { Sí } \\
\text { No }\end{array}$ & $\begin{array}{c}3 \\
144\end{array}$ & $\begin{array}{l}2.0 \\
98\end{array}$ & $\begin{array}{c}12 \\
853\end{array}$ & $\begin{array}{c}1.4 \\
98.6\end{array}$ & $1.48(0.41-5.31)$ & 0.375 \\
\hline
\end{tabular}

EPOC = enfermedad pulmonar obstructiva crónica, RM =razón de momios, IC 95 \% = intervalo de confianza de 95 \%.

Tabla 3. Determinantes sociales de la salud, comorbilidades de pacientes con COVID-19 agrupados según la gravedad de la enfermedad

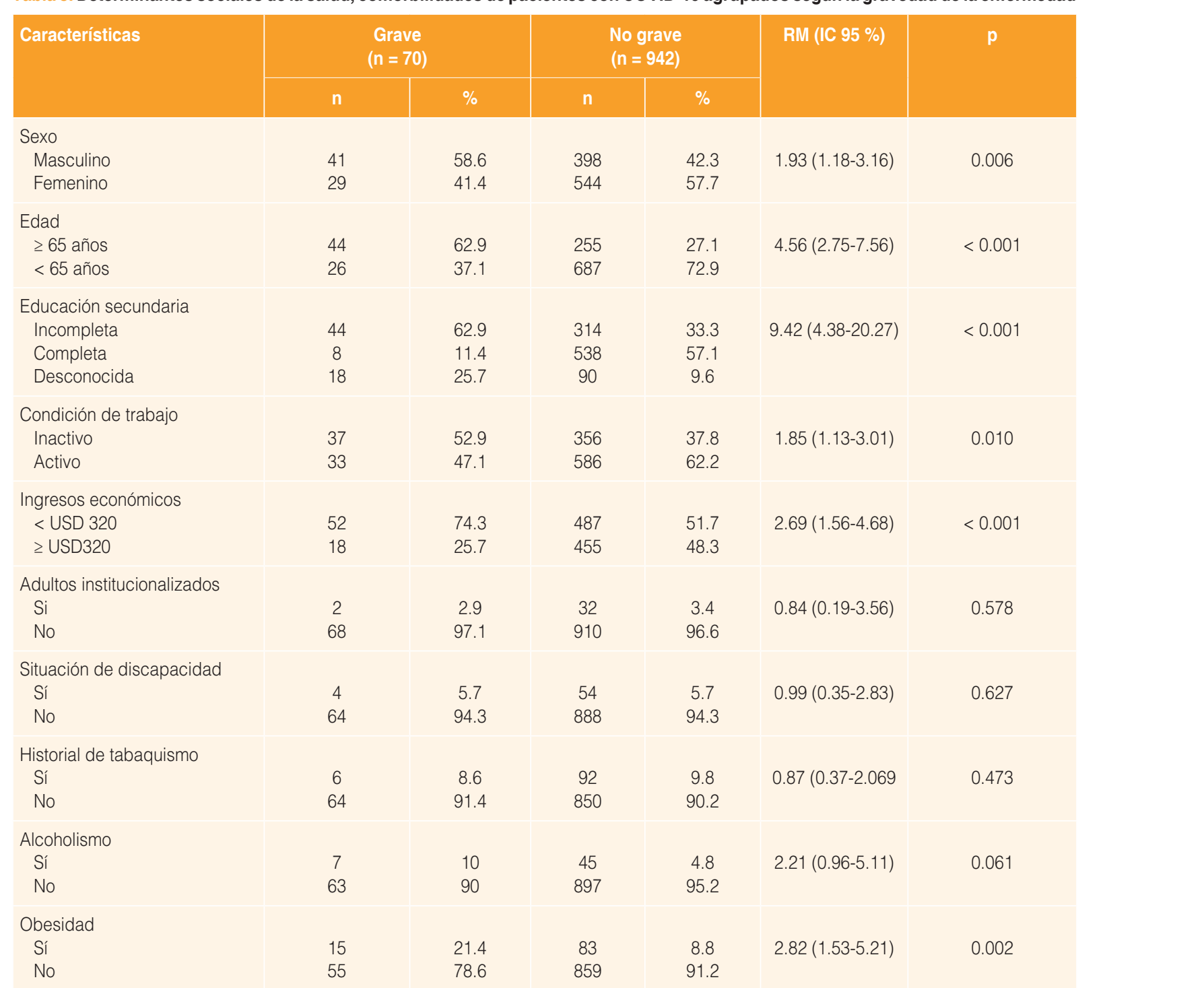




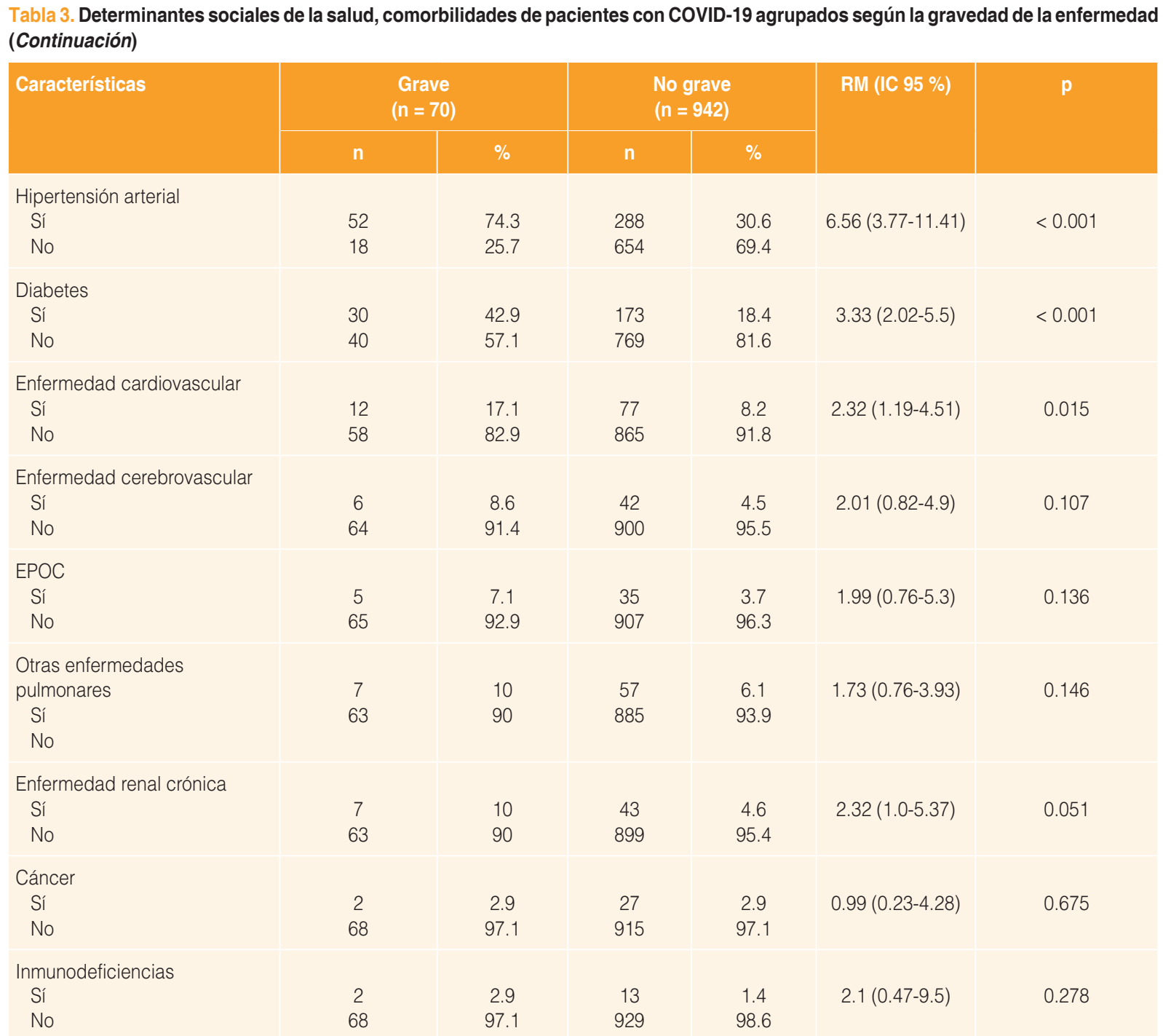

EPOC = enfermedad pulmonar obstructiva crónica, RM = razón de momios, IC $95 \%$ = intervalo de confianza de 95\%.

una relación con la gravedad de COVID-19; $6.9 \%$ de los pacientes fue ingresado a la UCl.

En cuanto a la edad, los adultos $\geq 65$ años presentaron un riesgo de mortalidad 24 veces mayor en comparación con los sujetos más jóvenes; esta asociación fue mucho mayor que la reportada por otros autores. ${ }^{24,25}$ Estas diferencias pueden ser explicadas por la relación entre los DSS y la mortalidad en adultos mayores en diferentes niveles, desde estilos de vida hasta macrodeterminantes socioeconómicos (dificultades económicas, nivel educacional). Por ejemplo, la evidencia muestra que los adultos mayores constituyen el grupo de edad más sedentario, ${ }^{26} \mathrm{a}$ su vez, la vida sedentaria se asocia a aumento en la mortalidad, desarrollo de enfermedades crónicas ${ }^{27} \mathrm{e}$ incremento de otros factores de riesgo que impactan en el nivel de gravedad de COVID-19 (por ejemplo, la hipertensión). ${ }^{25}$ Además, los ingresos económicos y el nivel educacional son factores que también están asociados a mayor mortalidad en personas mayores, ${ }^{13}$ lo cual es consistente con nuestros hallazgos. Otro grupo susceptible de fallecer a causa de COVID-19 son los adultos institucionalizados. La población que reside en centros de cuidados de larga estadía corresponde generalmente a adultos mayores que padecen múltiples enfermedades. ${ }^{28}$

En cuanto al nivel socioeconómico, nuestros resultados son similares a los reportados por otros autores. Ramírez y Lee ${ }^{29}$ encontraron que los DSS como la pobreza y el desempleo se asociaron positiva y significativamente a una mayor tasa de letalidad por COVID-19 en personas mayores. Estos hallazgos se 
explican por una mayor prevalencia de pobreza y desempleo según las diferentes áreas geográficas. Chile ha sido identificado como uno de los países con mayor desigualdad de ingresos, según la Organización para la Cooperación y Desarrollo Económico,$^{30}$ donde la segregación económica (expresada por los ingresos) se asocia a tasas más altas de mortalidad. ${ }^{31}$ Estos hallazgos refuerzan la idea de considerar a los DSS como una prioridad para la salud pública, con esfuerzos políticos hacia la reducción de la inequidad en salud para las generaciones futuras.

En relación con las personas en situación de discapacidad, en este grupo observamos una mayor asociación con la mortalidad. Las personas en situación de discapacidad tienen una mayor probabilidad de obtener resultados en salud por debajo de lo esperado, particularmente en países de bajos y medianos ingresos. ${ }^{32,33}$ La discapacidad está relacionada con otros DSS (como el desempleo) ${ }^{34} y$ otras condiciones de salud que aumentan la tasa de mortalidad por COVID-19, por ejemplo, diabetes y enfermedad cardiovascular. ${ }^{33}$ Nuestros hallazgos muestran la necesidad urgente de mejorar la prestación de atención médica a este grupo.

Respecto de las comorbilidades, la hipertensión fue una comorbilidad reportada frecuentemente en nuestra población, con una asociación significativa con la mortalidad y gravedad de COVID-19, similar a lo informado por otros autores. ${ }^{1,35}$ Aunque las comorbilidades de riesgo para el agravamiento o muerte por COVID19 se han descrito ampliamente en la literatura, con mayor frecuencia las personas con un nivel socioeconómico más bajo presentan estos factores de riesgo, incluyendo hipertensión, diabetes, obesidad, enfermedades cardiacas y enfermedades respiratorias crónicas. ${ }^{6,36}$ Por tanto, no podemos descartar que la fuerza de asociación entre estas variables también está relacionada con los DSS en nuestra población. Por ejemplo, la hipertensión es la condición responsable de la mayor carga de enfermedad en Chile, que afecta a una mayor proporción de personas con bajo nivel educativo y adultos mayores. ${ }^{37}$ Este problema de salud pública en Chile se ha hecho más evidente con la pandemia, lo que demuestra la importancia de generar políticas públicas en salud que puedan prevenir la prevalencia de estos factores de riesgo.

Respecto a las futuras necesidades de los supervivientes de COVID-19, cabe señalar que las limitaciones para realizar ejercicio, junto con las secuelas físicas y psicológicas constituyen las principales consecuencias del daño pulmonar grave, ${ }^{38}$ por lo que un grupo importante de estos pacientes requerirá rehabilitación. ${ }^{39}$ Los DSS también están relacionados con el acceso y la participación en programas de rehabilitación. ${ }^{40}$ Por ejemplo, las dificultades económicas comprenden una barrera estructural que afecta la adherencia a la rehabilitación pulmonar. ${ }^{41}$ Los equipos multidisciplinarios de rehabilitación deben ser conscientes de las desigualdades en salud provocadas por Ios DSS y deben implementar estrategias especializadas para mejorar la funcionalidad a largo plazo de estos pacientes.

Una limitación de nuestro estudio fue el posible subregistro de las comorbilidades en la historia clínica de cada paciente, que podría subestimar la verdadera fuerza de la asociación con los resultados clínicos. Sin embargo, nuestros hallazgos fueron consistentes con los informados en la literatura. También es importante mencionar que se aplican solo a la realidad de un país, por lo tanto se requieren investigaciones multicéntricas para confirmar estos hallazgos.

\section{Conclusiones}

Los DSS estructurales tuvieron una fuerte asociación con la mortalidad y severidad de COVID-19, en particular la edad, el nivel educativo, el desempleo y los ingresos económicos. Estos hallazgos son relevantes porque confirman que las condiciones socioeconómicas pueden influir tanto en la salud de la población como las características individuales de las personas. Es importante que los DSS se consideren una prioridad de salud pública y se centren los esfuerzos políticos en reducir las desigualdades en salud para las generaciones futuras.

\section{Conflicto de intereses}

Los autores declaran no tener conflicto de intereses alguno.

\section{Financiamiento}

La presente investigación no recibió ninguna beca específica de agencias de los sectores públicos, comercial o sin ánimo de lucro.

\section{Responsabilidades éticas}

Protección de personas y animales. Los autores declaran que los procedimientos seguidos se conformaron a las normas éticas del comité de 
experimentación humana responsable y de acuerdo con la Asociación Médica Mundial y la Declaración de Helsinki.

Confidencialidad de los datos. Los autores declaran que siguieron los protocolos de su centro de trabajo sobre la publicación de datos de pacientes.

Derecho a la privacidad y consentimiento informado. Los autores declaran que en este artículo no aparecen datos de pacientes.

\section{Bibliografía}

1. Zhou F, Yu T, Du R, Fan G, Liu Y, Xiang J, et al. Clinical course and risk factors for mortality of adult inpatients with COVID-19 in Wuhan, China: a retrospective cohort study. Lancet. 2020;395:1054-1062.

2. Adhikari SP, Meng S, Wu YJ, Mao YP, Ye RX, Wang QZ, et al. Epidemiology, causes, clinical manifestation and diagnosis, prevention and control of coronavirus disease (COVID-19) during the early outbreak period: a scoping review. Infect Dis Poverty. 2020;9:29.

3. Zhu N, Zhang D, Wang W, Li X, Yang B, Song J, et al. A novel coronavirus from patients with pneumonia in China, 2019. N Engl J Med. 2020;382:727-733.

4. Metlay JP, Waterer GW, Long AC, Anzueto A, Brozek J, Crothers K, et al. Diagnosis and treatment of adults with community-acquired pneumonia. An official clinical practice guideline of the American Thoracic Society and Infectious Diseases Society of America. Am J Respir Crit Care Med. 2019;200:e45-e67.

5. Gearhart AM, Furmanek S, English C, Ramírez J, Cavallazzi R. Predicting the need for ICU admission in community-acquired pneumonia. Respir Med. 2019;155:61-65.

6. Bambra C, Riordan R, Ford J, Matthews F. The COVID-19 pandemic and health inequalities. J Epidemiol Community Health. 2020;74:964-968.

7. Takian A, Kiani MM, Khanjankhani K. COVID-19 and the need to prioritize health equity and social determinants of health. Int J Public Health. 2020;65:521-523.

8. Marmot M, Friel S, Bell R, Houweling TAJ, Taylor S. Closing the gap in a generation: health equity through action on the social determinants of health. Lancet. 2008;372:1661-1669.

9. Marmot M. Social determinants of health inequalities. Lancet 2005;365:1099-1104.

10. Hernández LJ, Ocampo J, Ríos DS, Calderón C. The WHO model as a guideline for public health based on social determinants. Rev Salud Publica (Bogotá). 2017;19:393-395

11 Solar O, Irwin A. Social determinants, political contexts and civil society action: a historical perspective on the Commission on Social Determinants of Health. Health Promot J Austr. 2006:17:180-185.

12. De Lima-Silva V, Pessoa-Cesse EÂ, Pessoa-Militão-de Albuquerque MF. Social determinants of death among the elderly: a systematic literature review. Rev Bras Epidemiol. 2014;17:178-193.

13. Bassuk SS, Berkman LF, Amick BC. Socioeconomic status and mortality among the elderly: findings from four US communities. Am J Epidemiol. 2002;155:520-533.

14. Signorello LB, Cohen SS, Williams DR, Munro HM, Hargreaves MK, Blot WJ. Socioeconomic status, race, and mortality: a prospective cohort study. Am J Public Health. 2014;104:e98-e107.

15. Sooryanarain $\mathrm{H}$, Elankumaran $\mathrm{S}$. Environmental role in influenza virus outbreaks. Annu Rev Anim Biosci. 2015;3:347-373.

16. Berlin DA, Gulick RM, Martínez FJ. Severe Covid-19. N Engl J Med. 2020:383:2451-2460

17. Richardson S, Hirsch JS, Narasimhan M, Crawford JM, McGinn T, Davidson $\mathrm{KW}$, et al. Presenting characteristics, comorbidities, and outcomes among 5700 patients hospitalized with COVID-19 in the New York City area. JAMA. 2020:323:2052-2059.
18. Noriea AH, Patel FN, Werner DA, Peek ME. A narrative review of physician perspectives regarding the social and environmental determinants of obesity. Curr Diab Rep. 2018;18:24.

19. Summers JA, Stanley J, Baker MG, Wilson N. Risk factors for death from pandemic influenza in 1918-1919: a case-control study. Influenza Other Respir Viruses. 2014;8:329-338.

20. Huang C, Wang Y, Li X, Ren L, Zhao J, Hu Y, et al. Clinical features of patients infected with 2019 novel coronavirus in Wuhan, China. Lancet. 2020;395:497-506.

21. Chen $\mathrm{H}$, Cohen $\mathrm{P}$, Chen $\mathrm{S}$. How big is a big odds ratio? Interpreting the magnitudes of odds ratios in epidemiological studies. Communications in Statistics - Simulation and Computation. 2010;39:860-864.

22. Epidemiology Working Group for NCIP Epidemic Response, Chinese Center for Disease Control and Prevention. The epidemiological characteristics of an outbreak of 2019 novel coronavirus diseases (COVID-19) in China. Zhonghua Liu Xing Bing Xue Za Zhi. 2020;41:145-151.

23. Chen N, Zhou M, Dong X, Qu J, Gong F, Han Y, et al. Epidemiological and clinical characteristics of 99 cases of 2019 novel coronavirus pneumonia in Wuhan, China: a descriptive study. Lancet. 2020;395:507-513.

24. Du RH, Liang LR, Yang CQ, Wang W, Cao TZ, Li M, et al. Predictors of mortality for patients with COVID-19 pneumonia caused by SARS-CoV-2: a prospective cohort study. Eur Respir J. 2020:55:2000524.

25. Li X, Xu S, Yu M, Wang K, Tao Y, Zhou Y, et al. Risk factors for severity and mortality in adult COVID-19 inpatients in Wuhan. J Allergy Clin Immunol. 2020;146:110-118

26. Wullems JA, Verschueren SMP, Degens $\mathrm{H}$, Morse $\mathrm{Cl}$, Onambélé GL. A review of the assessment and prevalence of sedentarism in older adults, its physiology/health impact and non-exercise mobility counter-measures. Biogerontology. 2016;17:547-565.

27. Tremblay MS, Colley RC, Saunders TJ, Healy GN, Owen N. Physiological and health implications of a sedentary lifestyle. Appl Physiol Nutr Metab. 2010;35:725-740.

28. D'Adamo H, Yoshikawa T, Ouslander JG. Coronavirus disease 2019 in geriatrics and longtterm care: the ABCDs of COVID 019. J Am Geriatr Soc. 2020;68:912-917.

29. Ramírez IJ, Lee J. COVID-19 emergence and social and health determinants in Colorado: a rapid spatial analysis. Int $\mathrm{J}$ Environ Res Public Health. 2020;17:3856

30. Organisation for Economic Co-operation and Development [Internet]. Francia: Society at a Glance 2014; 2014.

31. Sánchez H, Albala C. Inequalities in health. Adult mortality in communities of Metropolitan Santiago. Rev Med Chil. 2004;132:453-460.

32. Armitage R, Nellums LB. The COVID-19 response must be disability inclusive. Lancet Public Health. 2020;5:e257.

33. The Lancet. Prioritising disability in universal health coverage. Lancet. 2019;394:187.

34. Bambra C, Pope D. What are the effects of anti-discriminatory legislation on socioeconomic inequalities in the employment consequences of ill health and disability? J Epidemiol Community Health. 2007;61:421-426.

35. Guan WJ, Liang WH, Zhao Y, Liang HR, Chen ZS, Li YM, et al. Comorbidity and its impact on 1590 patients with COVID-19 in China: a nationwide analysis. Eur Respir J. 2020;55:2000547.

36. Guo L, Wei D, Zhang X, Wu Y, Li Q, Zhou M, et al. Clinical features predicting mortality risk in patients with viral pneumonia: the MuLBSTA score. Front Microbiol. 2019;10:2752.

37. Ministerio de Salud. Encuesta Nacional de Salud 2009-2010. Chile: Ministerio de Salud; 2010.

38. Herridge MS, Tansey CM, Matté A, Tomlinson G, Díaz-Granados N, Cooper A, et al. Functional disability 5 years after acute respiratory distress syndrome. N Engl J Med. 2011;364:1293-1304.

39. Rivera-Lillo G, Torres-Castro R, Fregonezi G, Vilaró J, Puppo H. Challenge for rehabilitation after hospitalization for COVID-19. Arch Phys Med Rehabil. 2020:101:1470-1471.

40. Frier A, Barnett F, Devine S. The relationship between social determinants of health, and rehabilitation of neurological conditions: a systematic literature review. Disabil Rehabil. 2017:39:941-948.

41. Oates GR, Niranjan SJ, Ott C, Scarinci I, Schumann C, Parekh T, et al. Adherence to pulmonary rehabilitation in COPD: A qualitative exploration of patient perspectives on barriers and facilitators. J Cardiopulm Rehabil Prev. 2019:39:344-349. 